\title{
Linking chemical reaction intermediates of the click reaction to their molecular diffusivity
}

Tian Huang, ${ }^{a}$ Bo Li, ${ }^{a}$ Huan Wang, ${ }^{b^{*}}$ and Steve Granick ${ }^{a, c^{*}}$

${ }^{a}$ Center for Soft and Living Matter, Institute for Basic Science (IBS), Ulsan 44919, South Korea

${ }^{\mathrm{b}}$ College of Chemistry and Molecular Engineering, Peking University, Beijing, 100871, P. R. China

${ }^{\mathrm{c}}$ Departments of Chemistry and Physics, Ulsan National Institute of Science and Technology (UNIST), Ulsan 44919, South Korea

\begin{abstract}
$\underline{\text { Abstract }}$
Bipolar reactions have been provoked by reports of boosted diffusion during chemical and enzymatic reactions. To some, it is intuitively reasonable that relaxation to truly Brownian motion after passing an activation barrier can be slow, but to others the notion is so intuitively unphysical that they suspect the supporting experiments to be artifact. Here we study a chemical reaction according to whose mechanism some intermediate species should speed up while others slow down in predictable ways, if the boosted diffusion interpretation holds. Experimental artifacts would do not know organic chemistry mechanism, however. Accordingly, we scrutinize the absolute diffusion coefficient (D) during intermediate stages of the CuAAC reaction (coppercatalyzed azide-alkyne cycloaddition click reaction), using proton pulsed field-gradient nuclear magnetic resonance (PFG-NMR) to discriminate between the diffusion of various reaction intermediates. For the azide reactant, its $\mathrm{D}$ increases during reaction, peaks at the same time as peak reaction rate, then returns to its initial value. For the alkyne reagent, its D decreases consistent with presence of the intermediate large complexes formed from copper catalyst and its ligand, except for the $2 \mathrm{Cu}$-alk complex whose more rapid $\mathrm{D}$ may signify that this species is the
\end{abstract}


real reactive complex. For the product of this reaction, its D increases slowly as it detaches from the triazolide catalyst complex. These examples of enhanced diffusion for some molecular species and depressed diffusion for others causes us to conclude that diffusion coefficients during these elementary reactions are influenced by two components: hydrodynamic radius increase from complex formation, which slows diffusion, and energy release rate during the chemical reaction, which speeds it up. We discuss possible mechanisms and highlight that too little is yet understood about slow solvent reorganization during chemical reactions. 
A chemical reaction frequently follows a reaction cycle with multiple intermediate steps during which both electronic and physical structure change successively. Such reaction cycles are ubiquitous in both synthetic chemistry and biochemistry. We are interested here in the click reaction $^{1-7}$, a group of versatile reactions that is exceptionally useful and simple to implement with high product yield. Recently we reported changes of molecular diffusivity during several common chemical reactions including the click reaction based on two independent experimental methods, a microfluidics design and pulsed field-gradient $\mathrm{NMR}^{8-9}$, but in that study the intermediate reaction steps ${ }^{6-7}$ were not considered and our analysis involved normalized data rather than its absolute values. Here, inspecting a wider range of reagent stoichiometry, we focus on absolute values of diffusion coefficient and on how the mobility of intermediates in the catalytic cycle correlates with the extent of reaction. The findings highlight the twin influences of hydrodynamic radius changes, and reaction-induced boosted diffusion.

We vary the reactant stoichiometric ratio as this highlights the respective influence of each reactant on diffusion during intermediate steps of the reaction. The net reaction that describes the CuAAC reaction, copper-catalyzed azide-alkyne cycloaddition (Fig. 1A), has seen much work devoted in recent years to isolate intermediate products ${ }^{10-11}$. At the start, $\mathrm{Cu}(\mathrm{II})$ ion is reduced to $\mathrm{Cu}(\mathrm{I})$ by ascorbic acid. Alkyne reagent complexes with $\left[\mathrm{CuL}_{n}\right]$, then subsequently with another $\left[\mathrm{CuL}_{n}\right]$, where the notation $\mathrm{L}$ denotes a variety of possible ligands and the literature considers water, ascorbate, and other alkyne molecules to be candidates. The ligand complex is proposed to rearrange rapidly ${ }^{12}$. The terminal alkyne proton is present in the first two of these intermediates but not in the third, which means that NMR can discriminate them. These elementary steps are summarized schematically in Fig. 1B. 
Our experimental methods are described in Supporting Information (SI). Proton NMR spectra illustrated in Fig. S1 allow several intermediate steps to be discriminated. Because the intermediate $2 \mathrm{Cu}$-alk (dicopper complex) has methylene groups (2 protons) but no terminal proton ( 1 proton), the intensity ratio of methylene and terminal proton is not exactly 2 as expected from the pristine alkyne, but changes with time during reaction. Notably, the intensity ratio of methylene and terminal alkyne proton takes a minimum when the reaction rate peaks, as shown in Fig. 1C for a stoichiometry with azide reagent in excess which means that the faster the consumption of $2 \mathrm{Cu}$-alk, the faster the reaction rate. Corresponding to these elementary steps, Fig. 1D plots the time-dependent molar concentrations obtained by integrating the proton peaks. The findings are consistent when alkyne reactant is present in excess (Figs. S2A and S2B).

We employ pulsed field-gradient NMR to measure diffusion of these species and select catalyst and reagent concentrations to give reactions sufficiently slow to satisfy the quasi-steadystate approximation that concentration changes are negligible during the 3-5 min needed to measure each datum ${ }^{8-9}$. The methods that we use were challenged as artifact by authors who, in a Comment, made specific claims which alternative method is needed to avoid artifact ${ }^{13}$ but during this exchange of opinion we demonstrated the same findings regardless of which method was used $^{14}$. Our longer paper explaining this ${ }^{9}$ was followed by a second Comment by the same authors ${ }^{15}$ and our reply concluded that there is nothing really to argue about because everyone's online-deposited raw data is consistent ${ }^{16}$. In the present new study concerning intermediates, complexation with copper catalyst causes larger molar mass and consequently larger hydrodynamic volume, giving progressively slower diffusion coefficients of alkyne protons 2 and 1 (Fig. 1E). The molar mass of pristine alkyne, $\mathrm{Cu}$-alk, and 2Cu-alk are 56, 120 and $182 \mathrm{~g}$ $\mathrm{mol}^{-1}$, respectively. From this, the effective radius $\mathrm{R} \approx 0.28,0.36$ and $0.42 \mathrm{~nm}$, respectively is 
estimated (cube root of the molar volume implied by the molar mass and density $0.972 \mathrm{~g}-\mathrm{cm}^{-3}$ ). From the Stokes-Einstein equation, $\mathrm{D}=\mathrm{k}_{\mathrm{B}} \mathrm{T} / 6 \pi \eta \mathrm{R}$, the inverse dependence on $\mathrm{R}$ then predicts reduced diffusion coefficients 650 and $560 \mu^{2}-\mathrm{s}^{-1}$ for the $\mathrm{Cu}$-alk and $2 \mathrm{Cu}$-alk complexes respectively. While these estimates are qualitative as they ignore solvation and approximate complex molecular shapes as a single number, the radius, it is evident that there must be a trend towards slower diffusion owing to larger size. The apparent diffusion coefficient measured during the reaction is considered to be $\mathrm{D}$ of each of these species weighted by its molar concentration.

The NMR experiments give the time-dependent concentrations (Fig. 1D). They also give

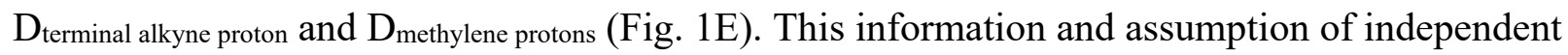
variables (reasonable because the overall concentration of all species is low) specifies $\mathrm{D}_{2 \mathrm{Cu} \text {-alk }}$. The argument yields $\mathrm{D}_{2 \mathrm{Cu} \text {-alk }} \approx 1100 \mu \mathrm{m}^{2} \mathrm{~s}^{-1}$ regardless of whether azide or alkyne is in excess

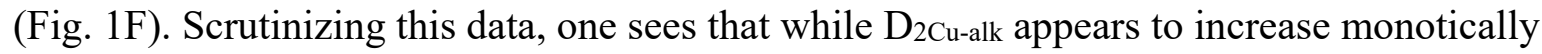
when azide is present in excess, there is a maximum when alkyne is in excess. The maximum $\mathrm{D}_{2 \mathrm{Cu} \text {-alk }}$ occurs at roughly the time when reaction rate is most rapid. The residual alkyne after reaction completion exists as a form of $2 \mathrm{Cu}$-alk; this complex is believed to experience fast degenerate rearrangement ${ }^{12}$. The baseline value of $2 \mathrm{Cu}$-alk varies according to the reactant stoichiometric ratio (Fig. S3A) because the ratio of alkyne and $\mathrm{Cu}$ in $2 \mathrm{Cu}$-alk complex can be different $^{17}$.

Similar estimates follow consistently from repeated experiments with reaction stoichiometry varied, though there is noise in this estimate because it depends on subtracting two large numbers. In fact, $\mathrm{D}$ of pristine alkyne without reaction, $\mathrm{D}=1060 \mathrm{\mu m}^{2} \mathrm{~s}^{-1}$, has nearly the 
same value. Our analysis of alkyne diffusion supersedes that in earlier papers from this laboratory in which consequences of complexation with the catalyst were not considered ${ }^{8-9}$, but the qualitative conclusion is unaffected. We conclude that $\mathrm{D}_{2 \mathrm{Cu} \text {-alk }}$ is more rapid than anticipated from its geometrical size.

We now consider azide, the second reagent, which according to the accepted reaction scheme ${ }^{7}$ adds to the reactive $2 \mathrm{Cu}$-alk complex. This is followed by several short-lived transition states, then a triazolide complex of product with $[\mathrm{CuLn}]$, and finally the pure reaction product. These elementary steps are summarized schematically in Fig. 2A. We mapped the concentrations of azide and product as a function of time under conditions of azide excess (Fig. 2B). In Fig. 2C, one sees that $\mathrm{D}$ of azide increases during reaction and reverts after reaction to its value in the absence of catalyst. Our finding that some species speed up during reaction while others slow down argue against the recent proposal ${ }^{18}$ that boosted diffusion is artifact because temperature or viscosity changes could explain the data. If this were so, all molecules would be affected uniformly.

Fig. 2E shows that the peak increase of $\mathrm{D}_{\text {azide }}$ was the same factor in this experiment as in our earlier experiments for which the reactant concentration was substantially higher (see figure caption). This peak coincides, in both instances, with the time when peak reaction rate was observed.

Boosted azide diffusion is also measured when alkyne is in stoichiometric excess (Fig. S3B). In this case, boosted diffusion persists during the reaction instead of falling back as shown for azide excess. Because the remaining small amounts of azide are all involved in the reaction near the reaction completion stage, it is reasonable that its diffusion remains boosted. Moreover, $\mathrm{D}_{\text {product }}$ increases with azide in excess (Fig. 2D). This is confirmed with alkyne in stoichiometric 
excess (Fig. S4A). Increase of $\mathrm{D}_{\text {product }}$ is expected from progressive dissociation of the product from the $\mathrm{Cu}$ catalyst. It is intriguing to notice that product dissociation occurs so slowly. The Droduct saturates roughly after passing the maximum reaction rate (Fig.S4B). This may be because protonation of triazole complex becomes more favorable when $2 \mathrm{Cu}$-alk forms by deprotonation.

Taken together, diffusion coefficients during this click chemical reaction appear to be influenced by two contributions: hydrodynamic radius increase from intermediates involving binding to the catalyst, which slows diffusion, and energy release rate during the chemical reaction, which speeds it up. The azide reactant diffuses more rapidly during reaction than in the absence of catalyst, under every reaction stoichiometry that we studied; this appears to be because the intermediate states in which it participates are too short-lived to be detectable by NMR, leaving only the influence of boosted diffusion. In fact, prior attempts by others to measure azide intermediates using mass spectrometry did not succeed even when using a ligand believed to prolong lifetime of those intermediate states ${ }^{19-20}$, which is consistent with these states having short lifetime. Copper-alkyne intermediates are longer-lived, however. They have been isolated $^{10-11}$ and characterized using mass spectrometry ${ }^{19-21}$. As estimated above, their radius in solution should increase, leading one to expect $\mathrm{D}$ in solution to decrease by this proportion, but quantitative consideration shows that the $2 \mathrm{Cu}$-alk complex diffuses more rapidly than expected from this argument. We interpret the difference to signify that this highly-reactive complex displays reaction-boosted diffusion.

What might be the physical mechanism? Too little is understood about intramolecular energy flow in which a fast, local component of intramolecular relaxation may be augmented by slower, possibly weaker relaxation ${ }^{22-23}$. Classical Marcus theory ${ }^{24-25}$ considers one-dimensional 
passage between two potential energy wells while all other degrees of freedom are considered, by assumption, to be a random thermal background; it does not deal with the energetics of solvent reorganization, which we consider likely to be the crux of this problem. The available spectroscopy experiments focus on using high-energy femtosecond pulses that probe transition states, while slower, lower-energy processes are not measured by the usual ultrafast techniques. However, restructuring of solvent molecules in response to dynamic changes of electric polarization has been noted repeatedly ${ }^{26-29}$. Progress in this area has been impeded by the paucity of experimental and simulation methods capable of exploring slower reorganization.

Our observation that enhanced diffusion correlates with reaction rate suggests that energy flow goes partly into chemical reaction, partly into translational diffusion, being coupled to the solvent. In fact, a growing body of literature considers solvent restructuring during chemical reactions. Orr-Ewing and coworkers have discussed these issues comprehensively ${ }^{30-33}$. In this spirit, organic chemists are well aware that reaction rates change according to the solvent ${ }^{33-35}$. New computational and experimental methods continue to be introduced ${ }^{36}$. On physical grounds, we consider it likely that chemical reactions produce electrified polarization of solvent molecules that solvate the molecules that react, thus providing a mechanism that funnels the rapid (fs) energy release of electronic rearrangement to slower, persistent reorganization of these larger and more diffuse solvation structures. Such reorganization proceeds in multiple steps, corresponding to multiple steps of electronic rearrangement, providing a possible mechanism by which one might expect the shapes of solvation shells to wiggle, wriggle and writhe. This could generate propulsion by a kind of swimming, a continuous slow action. Elsewhere we discussed that this could extend (for catalytic enzymes) for durations of a few microseconds by piconewton forces, dissipating energy of a few $k_{B} T$ as work against the viscous drag, times during which the 
boosted molecule maintain its general direction of motion until rotational diffusion randomizes its orientation ${ }^{37-38}$. Directed motion of this kind has been stated to be physically unrealistic because the skeptical authors imagined the force must be applied instantaneously ${ }^{18}$ but this we consider to be a red herring because in our scenario, force is applied persistently ${ }^{8,37-38}$. The same authors sought to argue reductio ad absurdum by pointing to the backward-forward chemical reactions that underlie an overall reversible chemical equilibrium ${ }^{18}$ but the click reaction is irreversible, so the argument appears to lack relevance. These considerations - local kick steps of boosted motion accompanied by reorientations from rotational Brownian diffusion -- would produce a random walk with effective diffusion coefficient larger than from just Brownian motion. The magnitude of augmentation would depend not only on the force released during each kick step but also on the kick frequency. Unfortunately, not enough is yet understood about how to define turnover time during catalytic cycles ${ }^{39}$.

In summary, this paper shows, as it considers the elementary steps of the catalytic reaction cycle, that the click chemical reaction is coupled to changes of molecular diffusivity. Some aspects are consistent with increased hydrodynamic radius owing to reaction intermediate states larger than the size of the neat reactants, while others show more rapid diffusivity. These findings point to the failure of the conventional Stoke-Einstein equation to fully explain diffusivity during chemical reaction. The magnitudes of the experimental enhancement we report are in the range also reported for catalytic enzymes ${ }^{37-38,40-43}$.

ACKNOWLEDGMENT. This study was supported by taxpayers of South Korea through the Institute for Basic Science (project code IBS-R020-D1). 


\section{Supporting Information}

Sample preparation. All reagents and solvent were used as received without further purification. Aqueous click reaction solutions containing alkyne (propargyl alcohol, 99\%, Sigma-Aldrich), azide (2-azidoacetic acid, Biosynth Carbosynth), (+)-sodium L-ascorbate $(\geq$ 98\%, Sigma-Aldrich) and copper (II) sulfate ( $\geq 99.99 \%$, Sigma-Aldrich) were freshly prepared before use. The desired amount of each reagent was added to $\mathrm{D}_{2} \mathrm{O}(99.9$ atom \% D) sequentially. In a typical procedure, the $2.7 \mu \mathrm{l}(75 \mathrm{mM})$ alkyne and $4.26 \mu \mathrm{l}(90 \mathrm{mM})$ azide were mixed at $600 \mu \mathrm{l}$ $\mathrm{D}_{2} \mathrm{O}, 5 \mathrm{mg}(40 \mathrm{mM})$ ascorbate was added and dissolved with vortex mixing, then we waited for $10 \mathrm{~min}$ to release heat. Then the reaction was initiated by adding $10.3 \mathrm{mM}$ copper (II) sulfate. The reaction solutions were mixed with vortex and filtered by $0.20 \mu \mathrm{m}$ membrane filter (PTFE, 4 mm Millex syringe filter) to remove the $\mathrm{Cu}$ nanoparticles produced by $\mathrm{Cu}(\mathrm{I})$ ions disproportionated reaction. Then the reaction solutions were transferred to standard $4.95 \mathrm{~mm}$ NMR tubes (Duran).

NMR experiments. ${ }^{1} \mathrm{H}$ NMR measurements were performed on a $600 \mathrm{MHz}$ FT-NMR spectrometer (Varian, VNMRS 600, Agilent) in the University Central Research Facility at UNIST (Ulsan National University of Science and Technology) at $25^{\circ} \mathrm{C}$. Typically, the first diffusion data is recorded at $15 \mathrm{~min}$, as a single measurement takes around $5 \mathrm{~min}$ depending on detailed experimental parameters, $5 \mathrm{~min}$ is needed to mix, filter, transfer and load the sample, another $5 \mathrm{~min}$ is needed prior to the first measurement to enable the standard alignment operations: lock, tune, shim, autogain. The spectra were obtained and processed using VnmrJ and MestReNova software. Having previously checked with care that findings do not depend on 
the sequence of applied magnetic field gradient ${ }^{14}$ we used the standard method of magnetic field gradients with increasing amplitude.

The double-stimulated echo convection suppression pulse, dstegp3s1d (Varian, $600 \mathrm{MHz}$ ), was used for diffusion measurement. The system-specific pulse duration $\delta$ was tuned between 500 $3000 \mu$ s with the diffusion time $\Delta=50 \mathrm{~ms}$, the pulse gradient ramped linearly from $5-95 \%$ of the maximum gradient $\left(50.35 \mathrm{G}-\mathrm{cm}^{-1}\right)$, typically in 16 steps to produce resulting intensity attenuation of the NMR peak of interest. The acquisition parameters for reactants were as follows, pulse duration $\delta=2000 \mu \mathrm{s}$, the diffusion time was $\Delta=50 \mathrm{~ms}$ and the relaxation delay time is $15 \mathrm{~s}$. For product, pulse duration $\delta=2500 \mu \mathrm{s}$, the diffusion time was $\Delta=50 \mathrm{~ms}$ and the relaxation delay time is $7 \mathrm{~s}$.

For intensity measurements at $4.1 \mathrm{ppm}$ and $2.9 \mathrm{ppm}$ of alkyne, $80 \mathrm{~s}$ relaxation delay time was used. From concern about potential interference, no extra paramagnetic ingredients such as gadolinium were added to facilitate relaxation for faster measurements.

Data analysis. In order to consider peak shifts during reaction and also to reduce potential errors from manual peak integration, the data were exported from MestreNova Software (Bruker) and analyzed by homebuilt Interactive Data Language (IDL) code. Generally, the position of peaks of interest peaks was located within a certain chemical shift range, and then the integration range of $0.01 \mathrm{ppm}$ was used to obtain the peak intensity. These automated methods are considered to be an improvement over the manual integration we used in earlier publications ${ }^{8-9}$. According to the Stejskal-Tanner equation, plotting the peak intensities against the calibrated gradient values with semi-log scales, the slope specifies diffusion coefficient in the conventional manner. 


\section{$\underline{\text { Figure Captions }}$}

Fig. 1. Elementary reactions during the first portion (reaction with alkyne reagent) of the click reaction cycle. (A) Net click reaction. The protons measured in this paper by pulsed fieldgradient NMR are indicated by numbers (1), (2), (3) and (5); ascorbate peaks (4) were reported previously $^{8}$. (B) Summary of elementary steps leading to reactive acetylide complex from alkyne and $\mathrm{Cu}(\mathrm{I})$ catalyst. (C) Reaction rate (right ordinate) and ratio of peak intensity of protons 2 and 1 (left ordinate) is plotted against time for alkyne:azide $=0.83(75: 90 \mathrm{mM})$. (D) Timedependent total alkyne concentration (species $\mathrm{i}+\mathrm{ii}+\mathrm{iii}$ indicated in panel B), concentration of neat alkyne and $\mathrm{Cu}$-alk ( $\mathrm{i}+\mathrm{ii}$ ), and concentration of $2 \mathrm{Cu}$-alk (iii), and product concentration, are plotted for the same reaction as panel C. (E) Diffusion coefficients are plotted against time for protons (1) and (2) with alkyne:azide $=0.83$ (left panel) and alkyne:azide $=1.67$ (right panel;

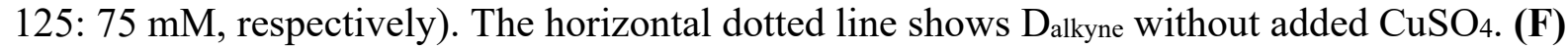

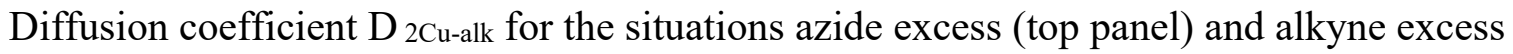
(bottom panel). Smaller symbols show 3 independent experiments. Larger circles are their average during the longer indicated reaction times. In the data panels, the time when the reaction completes is shown by a vertical arrow.

Fig. 2. Elementary reactions during which azide reactant adds to copper-alkyne complex, $2 \mathrm{Cu}-$ alk (A) Schematic summary of the elementary steps ending in product. (B) Time-dependent concentrations of azide and product for alkyne:azide $=0.83$, for the same reaction condition as in Fig. 1. (C) Time-dependent diffusion coefficient $\mathrm{D}_{\text {azide }}$ deduced from proton (3). The horizontal dotted line is $\mathrm{D}_{\text {azide }}$ without added $\mathrm{CuSO}_{4}$. (D) Time-dependent diffusion coefficient $\mathrm{D}_{\text {product }}$ deduced from proton (5). (E) Comparing boosted diffusion to reaction rate. Top panel: timedependent ratio, $\mathrm{D}_{\text {azide }}$ normalized to its value without added $\mathrm{CuSO}_{4}$, for the same reaction condition as in panel $\mathrm{C}$ (filled symbols) and for alkyne:azide $=0.83$ (250: $300 \mathrm{mM}$ ). Bottom panel: time-dependent reaction rate for the experiments reported in the top panel. Vertical arrows denote time when the reaction completes.

Fig. S1. Representative proton NMR spectra and peak assignments measured for the click reaction under conditions analyzed in Fig. 1C. Time-dependent peak shifts are evident from comparing data at different times (illustrated here at $30 \mathrm{~min}$ and $110 \mathrm{~min}$ ), but peak-shift analysis is beyond the scope of this paper.

Fig. S2. Experiments under the situation of azide excess (Fig. 1C and 1D) are confirmed under the situation of alkyne excess. (A) Reaction rate (right ordinate) and ratio of peak intensity of protons 2 and 1 (left ordinate) are plotted against time for the situation of alkyne:azide $=125: 75$ mM. (B) Time-dependent total alkyne concentration (species i + ii + iii indicated in panel B), 
concentration of neat alkyne and $\mathrm{Cu}$-alk $(\mathrm{i}+\mathrm{ii})$, and concentration of $2 \mathrm{Cu}$-alk (iii), and product concentration. In both panels, the time when the reaction completes is shown by a vertical arrow.

Fig.S3. Experiments under the situation of azide excess (Fig. 1) are confirmed under the situation of alkyne excess, alkyne:azide ratios $=82.5: 75,90: 75,97.5: 75$, and 125:75 mM. (A) Time-

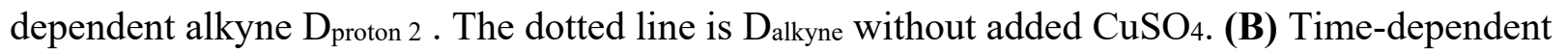
azide proton $\mathrm{D}_{\text {proton } 3}$ for these situations. The dotted line is $\mathrm{D}_{\text {azide }}$ without added $\mathrm{CuSO}_{4}$. Near the very end of reaction, the peak intensity of azide is too small to fit accurately, so we cannot measure $\mathrm{D}_{\text {azide }}$ up to the point of reaction completion. In both panels, the times when reaction completes are indicated by a vertical arrow.

Fig. S4. Experiments showing $D_{\text {product }}$ increase (Fig. 2D) are confirmed under the situation of alkyne excess with alkyne:azide ratios specified in the figure. Same situation as for Fig. S3. The times when reaction completes are indicated by a vertical arrow. 
Fig. 1

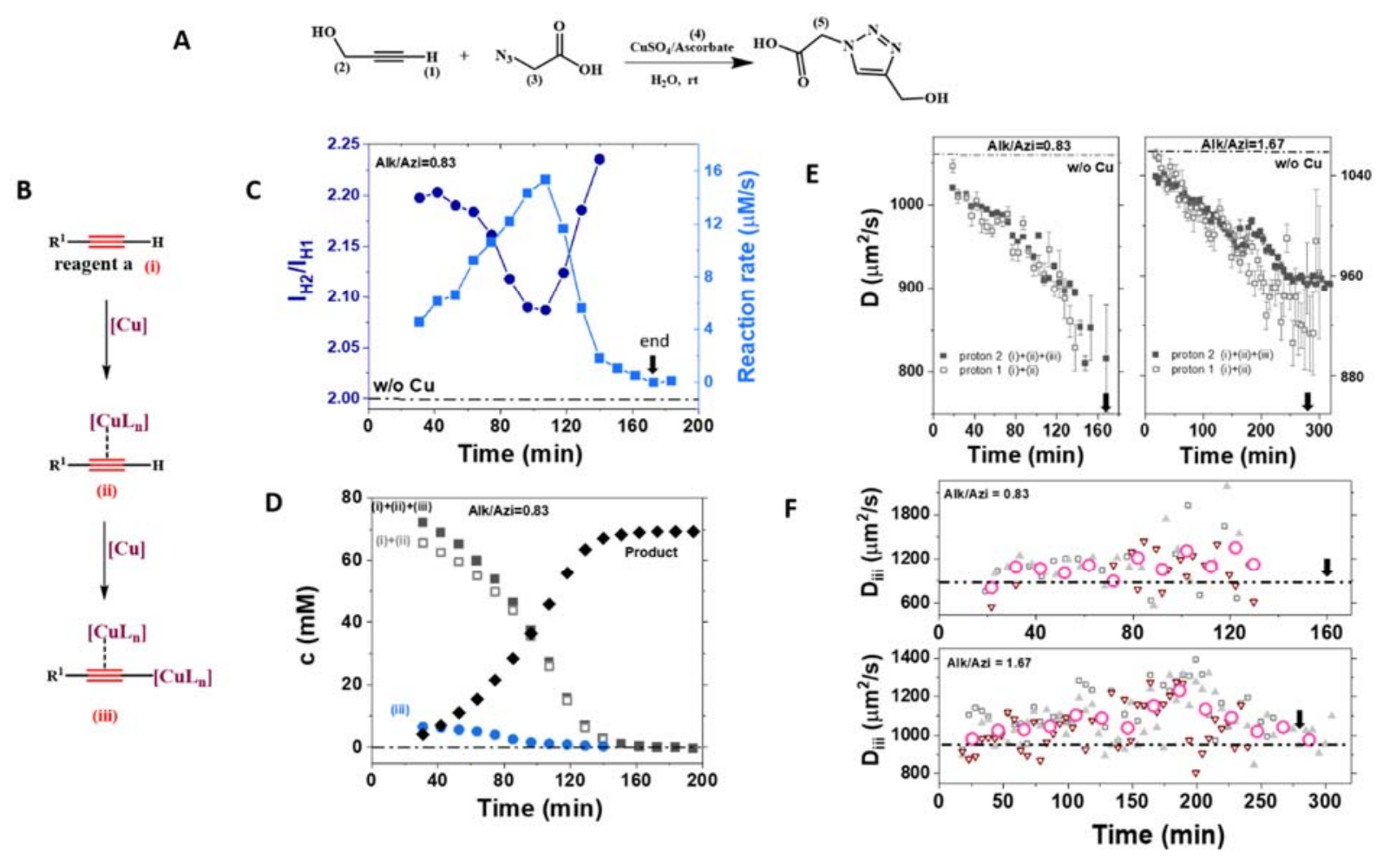


Fig. 2
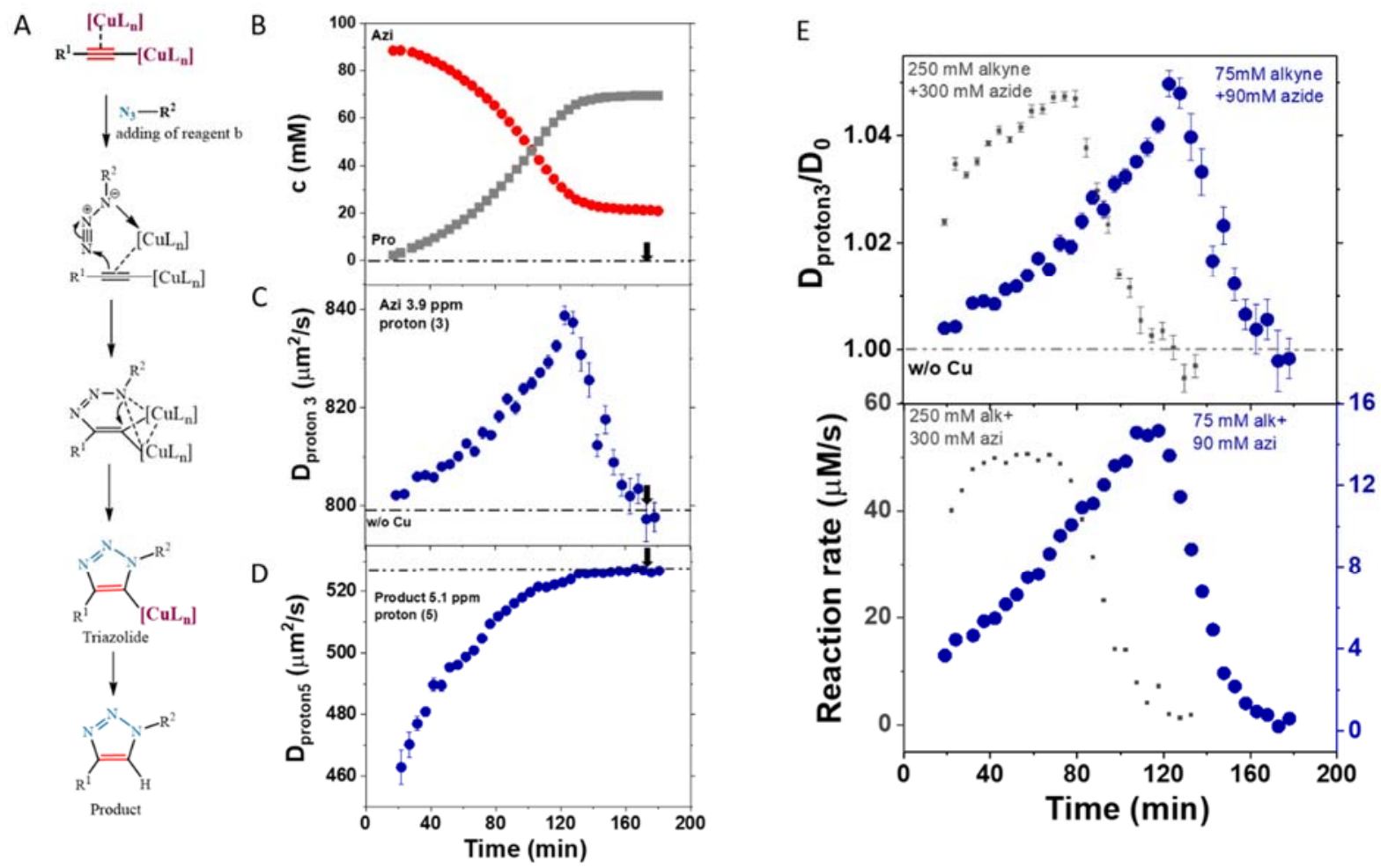
Fig. S1.

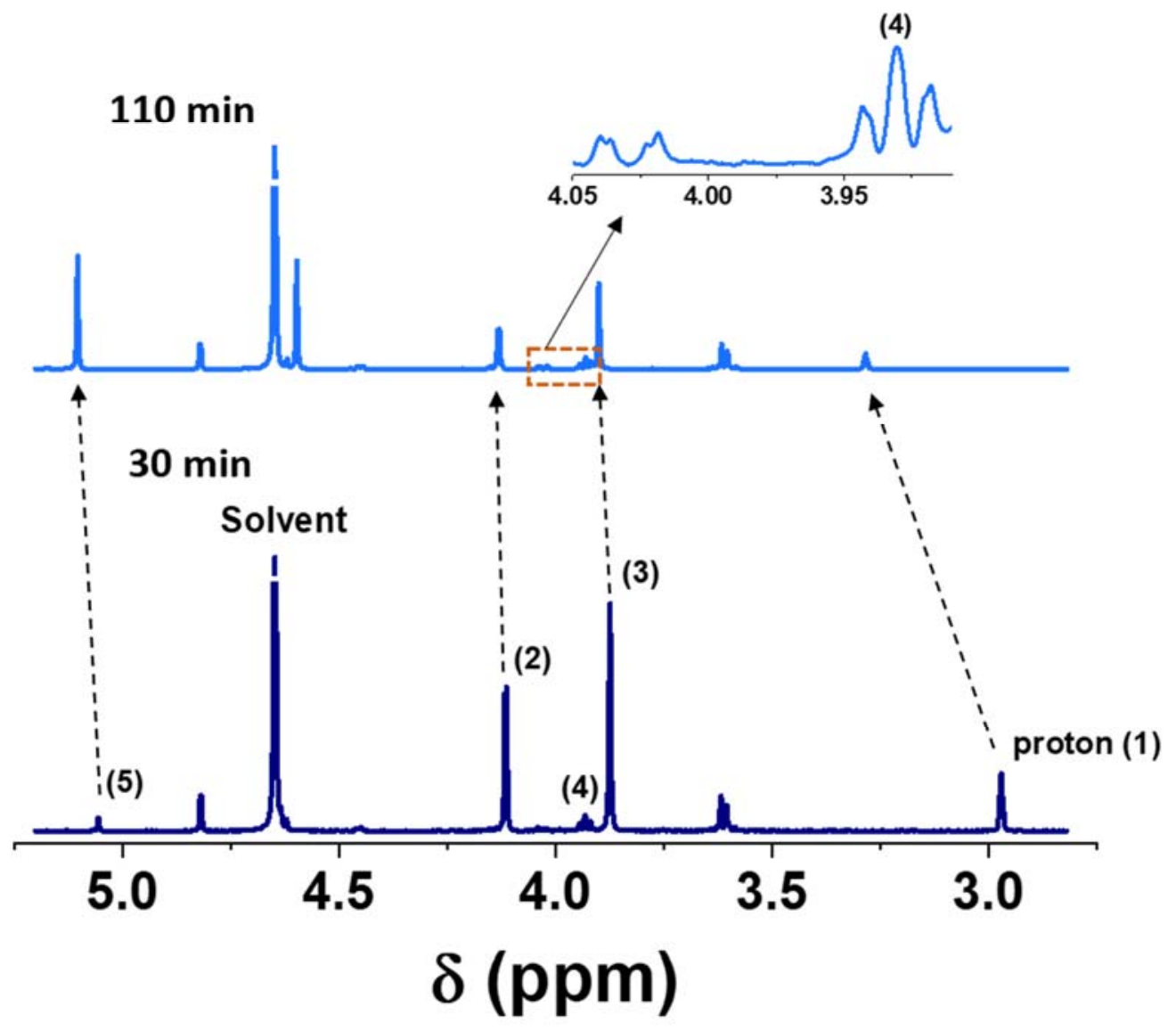


Fig. S2.
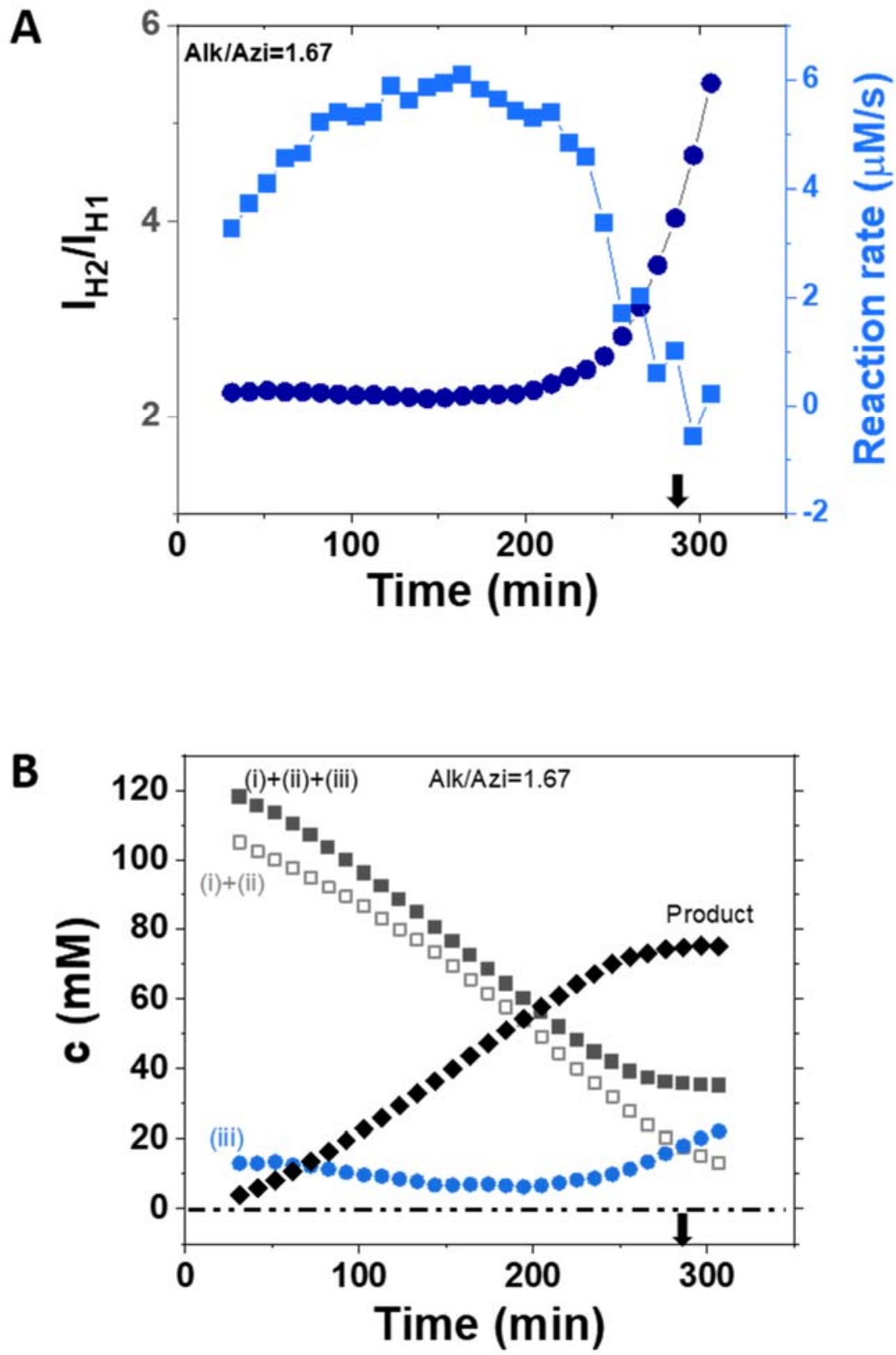
Fig. S3.

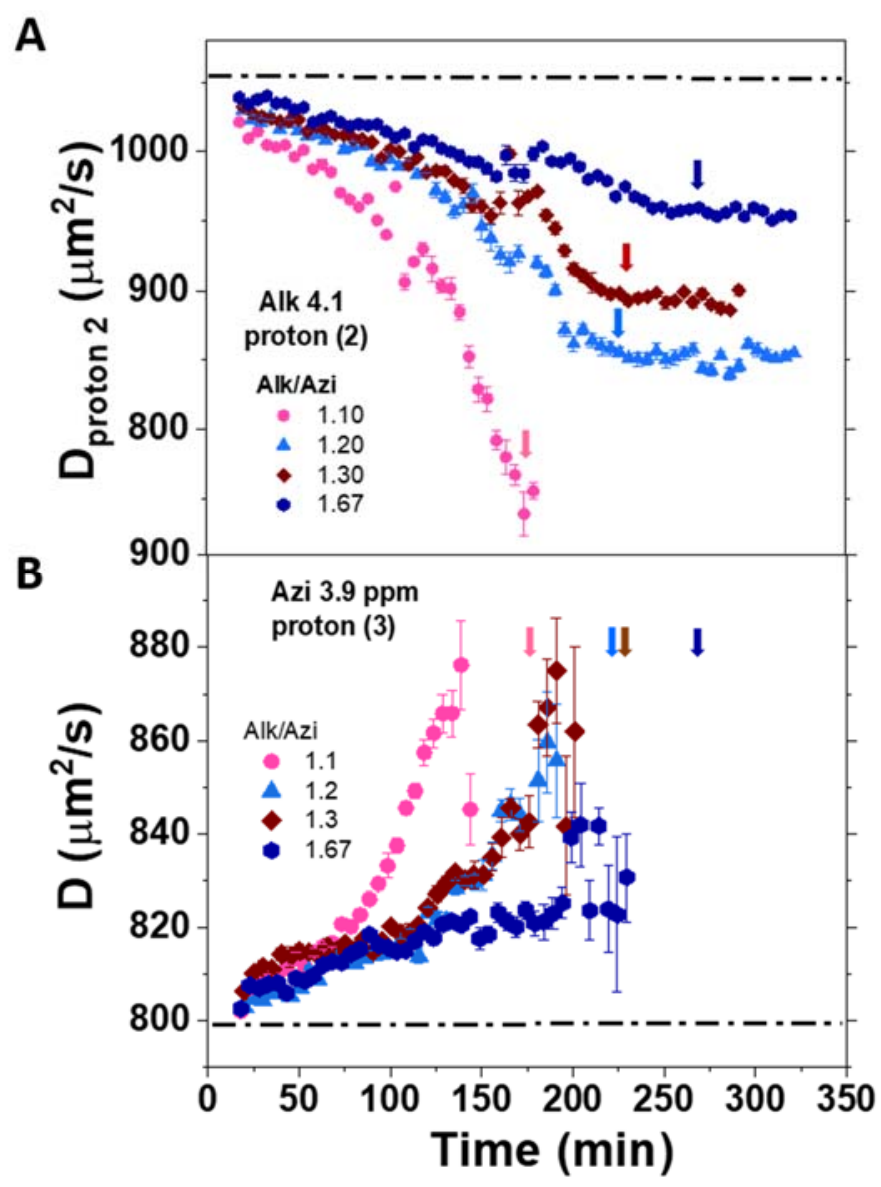


Fig. S4.

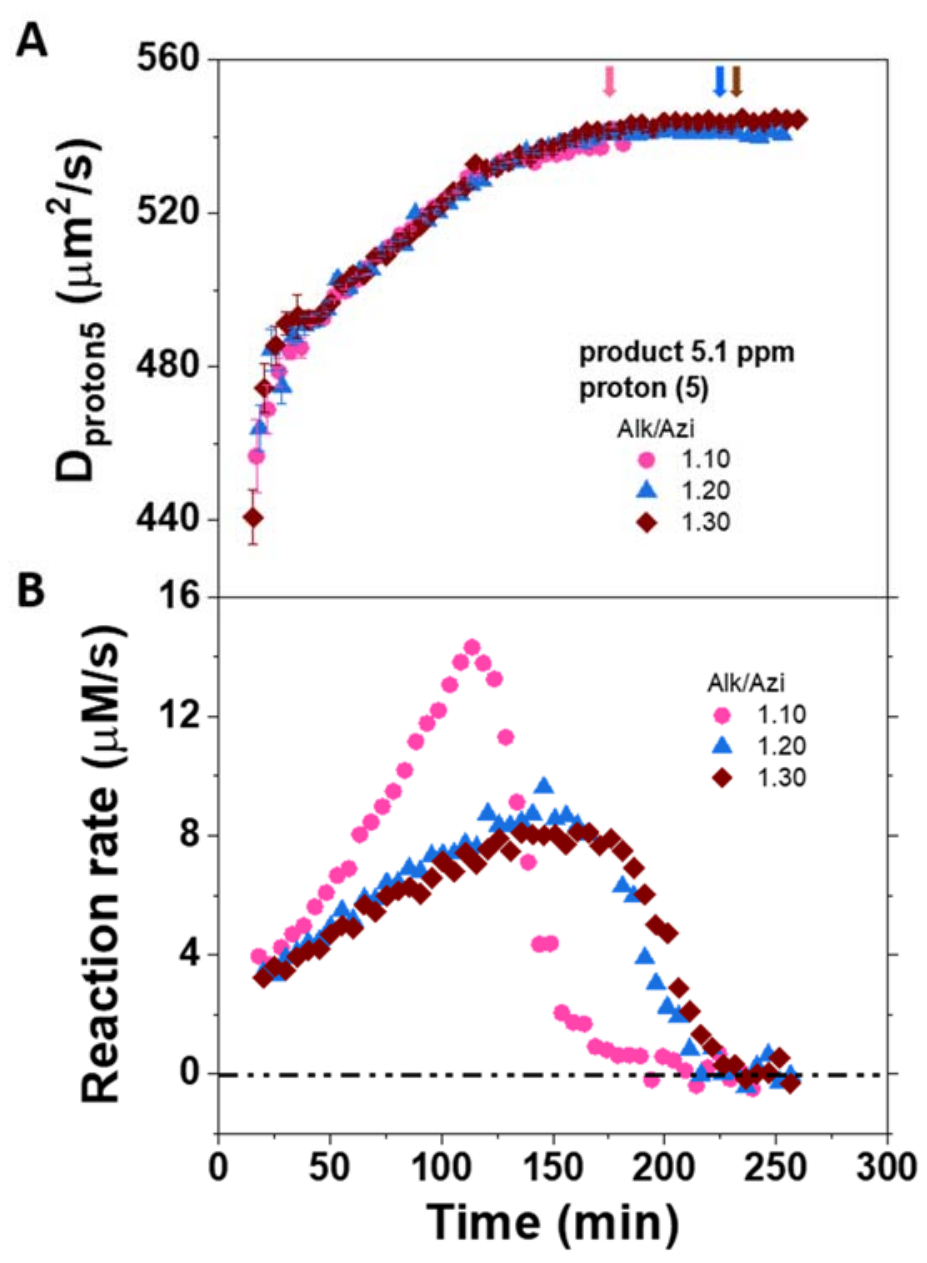




\section{$\underline{\text { References }}$}

1. Kolb, H. C.; Finn, M. G.; Sharpless, K. B., Click Chemistry: Diverse Chemical Function from a Few Good Reactions. Angewandte Chemie International Edition 2001, 40 (11), 2004-2021.

2. $\quad$ Meng, G.; Guo, T.; Ma, T.; Zhang, J.; Shen, Y.; Sharpless, K. B.; Dong, J., Modular click chemistry libraries for functional screens using a diazotizing reagent. Nature 2019, 574 (7776), 86-89.

3. Kolb, H. C.; Sharpless, K. B., The growing impact of click chemistry on drug discovery. Drug Discovery Today 2003, 8 (24), 1128-1137.

4. $\quad$ Moses, J. E.; Moorhouse, A. D., The growing applications of click chemistry. Chemical Society Reviews 2007, $36(8), 1249-1262$.

5. Hein, J. E.; Fokin, V. V., Copper-catalyzed azide-alkyne cycloaddition (CuAAC) and beyond: new reactivity of copper(i) acetylides. Chemical Society Reviews 2010, 39 (4), 1302-1315.

6. $\quad$ Himo, F.; Lovell, T.; Hilgraf, R.; Rostovtsev, V. V.; Noodleman, L.; Sharpless, K. B.; Fokin, V. V., Copper(I)-Catalyzed Synthesis of Azoles. DFT Study Predicts Unprecedented Reactivity and Intermediates. Journal of the American Chemical Society 2005, 127 (1), 210-216.

7. Worrell, B. T.; Malik, J. A.; Fokin, V. V., Direct Evidence of a Dinuclear Copper Intermediate in Cu(I)Catalyzed Azide-Alkyne Cycloadditions. Science 2013, 340 (6131), 457.

8. Wang, H.; Park, M.; Dong, R.; Kim, J.; Cho, Y.-K.; Tlusty, T.; Granick, S., Boosted molecular mobility during common chemical reactions. Science 2020, 369 (6503), 537.

9. Wang, H.; Huang, T.; Granick, S., Using NMR to Test Molecular Mobility during a Chemical Reaction. The Journal of Physical Chemistry Letters 2021, 12 (9), 2370-2375.

10. Nolte, C.; Mayer, P.; Straub, B. F., Isolation of a Copper(I) Triazolide: A “Click” Intermediate.

Angewandte Chemie International Edition 2007, 46 (12), 2101-2103.

11. Jin, L.; Tolentino, D. R.; Melaimi, M.; Bertrand, G., Isolation of bis(copper) key intermediates in Cucatalyzed azide-alkyne "click reaction". Science Advances 2015, 1 (5), e1500304.

12. Makarem, A.; Berg, R.; Rominger, F.; Straub, B. F., A Fluxional Copper Acetylide Cluster in CuAAC Catalysis. Angewandte Chemie International Edition 2015, 54 (25), 7431-7435.

13. Günther, J.-P.; Fillbrook, L. L.; MacDonald, T. S. C.; Majer, G.; Price, W. S.; Fischer, P.; Beves, J. E., Comment on "Boosted molecular mobility during common chemical reactions". Science 2021, 371 (6526), eabe8322.

14. Wang, H.; Park, M.; Dong, R.; Kim, J.; Cho, Y.-K.; Tlusty, T.; Granick, S., Response to Comment on "Boosted molecular mobility during common chemical reactions". Science 2021, 371 (6526), eabe8678.

15. Fillbrook L.; Günther, J.-P.; Majer, G.; Price, W. S.; Fischer, P.; Beves, J. E., Errors in the Use of NMR to Test Molecular Mobility during a Chemical Reaction. The Journal of Physical Chemistry Letters 2021, in press.

16. Huang T. , Wang H., Granick S., Reply to comment on 'Using NMR to Test Molecular Mobility during a Chemical Reaction'. The Journal of Physical Chemistry Letters 2021, In press.

17. Lang, H.; Jakob, A.; Milde, B., Copper(I) Alkyne and Alkynide Complexes. Organometallics 2012, 31 (22), 7661-7693.

18. Zhang, Y.; Hess, H., Chemically-powered swimming and diffusion in the microscopic world. Nature Reviews Chemistry 2021.

19. Iacobucci, C.; Reale, S.; Gal, J.-F.; De Angelis, F., Dinuclear Copper Intermediates in Copper(I)-Catalyzed Azide-Alkyne Cycloaddition Directly Observed by Electrospray Ionization Mass Spectrometry. Angewandte Chemie International Edition 2015, 54 (10), 3065-3068.

20. $\quad$ Chen, H.; Cai, C.; Li, S.; Ma, Y.; Luozhong, S.; Zhu, Z., Intermediates Stabilized by

Tris(triazolylmethyl)amines in the CuAAC Reaction. Chemistry - A European Journal 2017, 23 (19), 4730-4735.

21. He, Q.; Xing, Z.; Wei, C.; Fang, X.; Zhang, S.; Zhang, X., Rapid screening of copper intermediates in $\mathrm{Cu}(\mathrm{i})$-catalyzed azide-alkyne cycloaddition using a modified ICP-MS/MS platform. Chemical Communications 2016, 52 (69), 10501-10504.

22. Flowers, M. C.; Frey, H. M., Hot molecule effects in the thermal isomerization of methylbicyclo[2.1.0]pent-2-enes. Journal of the American Chemical Society 1972, 94 (24), 8636-8637.

23. Bragg, A. E.; Cavanagh, M. C.; Schwartz, B. J., Linear Response Breakdown in Solvation Dynamics Induced by Atomic Electron-Transfer Reactions. Science 2008, 321 (5897), 1817.

24. Gruebele, M.; Wolynes, P. G., Vibrational Energy Flow and Chemical Reactions. Accounts of Chemical Research 2004, 37 (4), 261-267.

25. Marcus, R. A.; Sutin, N., Electron transfers in chemistry and biology. Biochimica et Biophysica Acta (BBA) - Reviews on Bioenergetics 1985, 811 (3), 265-322. 
26. Jimenez, R.; Fleming, G. R.; Kumar, P. V.; Maroncelli, M., Femtosecond solvation dynamics of water. Nature 1994, 369 (6480), 471-473.

27. Hsu, C.-P.; Song, X.; Marcus, R. A., Time-Dependent Stokes Shift and Its Calculation from Solvent Dielectric Dispersion Data. The Journal of Physical Chemistry B 1997, 101 (14), 2546-2551.

28. Dinpajooh, M.; Newton, M. D.; Matyushov, D. V., Free energy functionals for polarization fluctuations: Pekar factor revisited. The Journal of Chemical Physics 2017, 146 (6), 064504.

29. Meng, X.; Guo, J.; Peng, J.; Chen, J.; Wang, Z.; Shi, J.-R.; Li, X.-Z.; Wang, E.-G.; Jiang, Y., Direct visualization of concerted proton tunnelling in a water nanocluster. Nature Physics 2015, 11 (3), 235-239.

30. Orr-Ewing, A. J., Perspective: Bimolecular chemical reaction dynamics in liquids. The Journal of Chemical Physics 2014, 140 (9), 090901.

31. Dunning, G. T.; Glowacki, D. R.; Preston, T. J.; Greaves, S. J.; Greetham, G. M.; Clark, I. P.; Towrie, M.; Harvey, J. N.; Orr-Ewing, A. J., Vibrational relaxation and microsolvation of DF after F-atom reactions in polar solvents. Science 2015, 347 (6221), 530.

32. Orr-Ewing, A. J., Taking the plunge: chemical reaction dynamics in liquids. Chemical Society Reviews 2017, 46 (24), 7597-7614.

33. Carpenter, B. K.; Harvey, J. N.; Orr-Ewing, A. J., The Study of Reactive Intermediates in Condensed Phases. Journal of the American Chemical Society 2016, 138 (14), 4695-4705.

34. Parker, A. J., Protic-dipolar aprotic solvent effects on rates of bimolecular reactions. Chemical Reviews 1969, 69 (1), 1-32.

35. Morris, W.; Lorance, E. D.; Gould, I. R., Understanding the Solvent Contribution to Chemical Reaction Barriers. The Journal of Physical Chemistry A 2019, 123 (49), 10490-10499.

36. Biasin, E.; Fox, Z. W.; Andersen, A.; Ledbetter, K.; Kjær, K. S.; Alonso-Mori, R.; Carlstad, J. M.; Chollet, M.; Gaynor, J. D.; Glownia, J. M.; Hong, K.; Kroll, T.; Lee, J. H.; Liekhus-Schmaltz, C.; Reinhard, M.; Sokaras, D.; Zhang, Y.; Doumy, G.; March, A. M.; Southworth, S. H.; Mukamel, S.; Gaffney, K. J.; Schoenlein, R. W.; Govind, N.; Cordones, A. A.; Khalil, M., Direct observation of coherent femtosecond solvent reorganization coupled to intramolecular electron transfer. Nature Chemistry 2021, 13 (4), 343-349.

37. Jee, A.-Y.; Cho, Y.-K.; Granick, S.; Tlusty, T., Catalytic enzymes are active matter. Proceedings of the National Academy of Sciences 2018, 115 (46), E10812.

38. Jee, A.-Y.; Dutta, S.; Cho, Y.-K.; Tlusty, T.; Granick, S., Enzyme leaps fuel antichemotaxis. Proceedings of the National Academy of Sciences 2018, 115 (1), 14.

39. Kozuch, S.; Martin, J. M. L., "Turning Over" Definitions in Catalytic Cycles. ACS Catalysis 2012, 2 (12), 2787-2794.

40. Muddana, H. S.; Sengupta, S.; Mallouk, T. E.; Sen, A.; Butler, P. J., Substrate Catalysis Enhances SingleEnzyme Diffusion. Journal of the American Chemical Society 2010, 132 (7), 2110-2111.

41. $\quad$ Sengupta, S.; Dey, K. K.; Muddana, H. S.; Tabouillot, T.; Ibele, M. E.; Butler, P. J.; Sen, A., Enzyme Molecules as Nanomotors. Journal of the American Chemical Society 2013, 135 (4), 1406-1414.

42. Dey, K. K.; Zhao, X.; Tansi, B. M.; Méndez-Ortiz, W. J.; Córdova-Figueroa, U. M.; Golestanian, R.; Sen, A., Micromotors Powered by Enzyme Catalysis. Nano Letters 2015, 15 (12), 8311-8315.

43. Jee, A.-Y.; Tlusty, T.; Granick, S., Master curve of boosted diffusion for 10 catalytic enzymes. Proceedings of the National Academy of Sciences 2020, 117 (47), 29435. 